Research Article

\title{
Classification of Material Type from Optical Coherence Tomography Images Using Deep Learning
}

\author{
Metin Sabuncu $\mathbb{i D}^{1}$ and Hakan Ozdemir (iD $^{2}$ \\ ${ }^{1}$ Department of Electrical and Electronics Engineering, Dokuz Eylül University, Izmir 35160, Turkey \\ ${ }^{2}$ Department of Textile Engineering, Dokuz Eylül University, Izmir 35160, Turkey \\ Correspondence should be addressed to Metin Sabuncu; metin.sabuncu@deu.edu.tr
}

Received 8 June 2021; Revised 27 September 2021; Accepted 5 October 2021; Published 20 October 2021

Academic Editor: Stefan Wabnitz

Copyright ( $) 2021$ Metin Sabuncu and Hakan Ozdemir. This is an open access article distributed under the Creative Commons Attribution License, which permits unrestricted use, distribution, and reproduction in any medium, provided the original work is properly cited.

\begin{abstract}
Classification of material type is crucial in the recycling industry since good quality recycling depends on the successful sorting of various materials. In textiles, the most commonly used fiber material types are wool, cotton, and polyester. When recycling fabrics, it is critical to identify and sort various fiber types quickly and correctly. The standard method of determining fabric fiber material type is the burn test followed by a microscopic examination. This traditional method is destructive, tedious, and slow since it involves cutting, burning, and examining the yarn of the fabric. We demonstrate that the identification procedure can be done nondestructively using optical coherence tomography (OCT) and deep learning. The OCT image scans of fabrics that are composed of different fiber material types such as wool, cotton, and polyester are used to train a deep neural network. We present the results of the created deep learning models' capability to classify fabric fiber material types. We conclude that fiber material types can be identified nondestructively with high precision and recall by OCT imaging and deep learning. Because classification of material type can be performed by OCT and deep learning, this novel technique can be employed in recycling plants in sorting wool, cotton, and polyester fabrics automatically.
\end{abstract}

\section{Introduction}

To know the fiber content of a fabric is essential because fiber content directly determines the performance, usage, and care of the fabric [1-3]. Due to their properties, cotton, wool, and polyester are the most widely used fibers in the textile industry [4]. Cotton is a natural plant-based fiber that is strong, absorbent, comfortable, and versatile [5]. It can shrink easily unless treated and is prone to wrinkling [6]. Wool is a natural animal-based fiber that is wrinkle resistant and possesses good insulation properties [7]. Wool is therefore a natural choice when making sweaters, socks, sportswear, dresses, and blankets [8]. Polyester is a synthetic man-made fiber type [9]. It is strong and elastic and collects static electricity [10]. Polyester has excellent wash and wear performance.

Traditionally, in order to determine the fiber content of a fabric, one performs a variety of different tests such as the burning test followed by inspecting the longitudinal and cross section of the fiber and a qualitative chemical analysis [11]. These methods are tedious, time consuming, and destructive and cannot be easily automated [12]. Newer methods use spectroscopy $[13,14]$, machine vision $[15,16]$, FTIR reflectance $[17,18]$, texture feature extraction [19], and computer-based algorithms for fiber identification. Especially in large-scale fabric separation and sorting, it is necessary to have a rapid, dependable, and automated method that classifies fiber content accurately [20]. The weaving and textile recycling industries require automated and reliable methods that can sort various fabric types efficiently [21].

In this study, we address the task of determining the fiber content of fabrics from a photonic perspective and present a novel technique. We, namely, make use of optical coherence tomography (OCT) imaging modality combined with deep learning. The OCT system employs an infrared light beam to 
obtain photonic depth images of the fabric in a contactless and nondestructive manner [22]. Because of its unique properties, OCT has been successfully applied to a variety of problems encountered in many different industries. OCT is suitable to use in inspection systems since it allows taking sensitive and high-resolution scans in a contactless and nondestructive fashion [23]. For example, OCT can be used to inspect defects on LCDs [24] and measure coating thicknesses on printed circuit boards [25]. In the textile industry, OCT was used to automatically classify weave patterns of fabrics [26-28]. Very recently, OCT was applied to the contactless measurement of fabric thickness [29]. Deep learning, as a novel method, has also been recently utilized in the automation and textile industry, especially in material [30] and fabric pattern recognition tasks [31-33]. Some possible applications of AI are discussed in detail in [34].

In this study, we utilized the photonic imaging modality of OCT, combined with automated deep learning, in order to determine the fiber content of woven fabric. The idea behind automated deep learning is letting a neural network design another neural network via neural architecture search [35]. Normally, it is very time consuming and tedious to design and choose the most suitable deep learning architecture. Reinforcement learning algorithms can make this process much quicker and smoother by enabling neural architecture search, hence automating machine learning. In this article, automated deep learning models were created that successfully identified fiber material types from OCT images. Our work involved taking OCT scans over fabrics composed solely of cotton, wool, and polyester. These scans were fed into a neural architecture search framework in order to accomplish fiber type identification from OCT images with automated deep learning.

\section{Materials and Methods}

The experimental setup is schematically illustrated in Figure 1. A laser diode with central wavelength at $930 \mathrm{~nm}$ generates broadband photons that allow for speckle-free imaging [36, 37]. The fabric samples to be measured are placed in the sample arm. The photons reflect from the sample surface and interfere in the fiber coupler with the beam coming from the reference arm. The one-dimensional OCT-A scan of the sample fabrics can be obtained by applying a Fourier transform to the signal falling onto the CCD camera. The two-dimensional OCT-B scans are obtained by scanning the light beam over the surface of the sample and adding corresponding OCT-A images sequentially. In this study, all OCT scans correspond to the OCT-B images of the fabric samples [27]. Before the OCT scans are performed, the burn test and microscopic viewing initially determined the fiber contents of the fabrics. The fabrics that consisted of only wool, cotton, or polyester were kept. For each fiber type, 3 different fabrics were arranged giving a total number of 9 separate samples. Each sample was put into the sample arm and measured by the OCT modality individually. In order to have a successful training, the deep learning algorithm requires at least 100 images per class. We therefore took

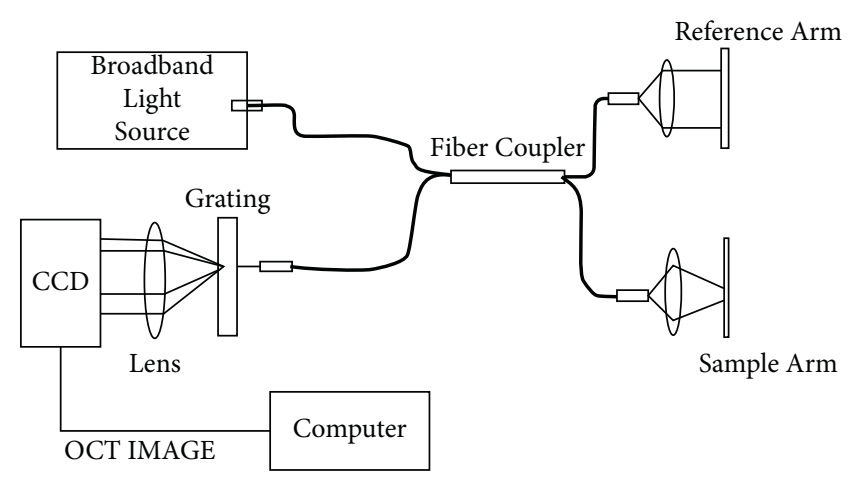

FIgURE 1: Diagram of the experimental setup. A broadband light source is coupled with a fiber and sent to the setup that, with the use of an optical grating and CCD, takes the spectral domain OCT scans. The material is placed in the sample arm. The OCT scans of fabric samples are recorded on the computer.

between 120 and 200 OCT scans per fabric. To have roughly uniform data across samples, the scans were fixed at $2 \mathrm{~mm}$ for each image and recorded in a portable network graphics format $[38,39]$. The OCT scans are raw OCT-B images saved through the image capture software program, and no extra image processing or filtering was performed.

The fabric data were separated into three categories. Groups 1, 2, and 3 consisted of fabrics made out of only cotton, wool, and polyester, respectively. These were placed in folders that became the labeled dataset for the classes in the deep learning training. In order to initiate the training, the dataset was uploaded onto the Cloud platform [35]. All the data are uploaded with this article and can be downloaded from the data repository [40].

The flow of the procedure for the automated deep learning application for fiber type identification is given in Figure 2. A commercial application programming interface is used to create and train the high-quality automated deep learning model via transfer learning [41]. To perform the classification task, the program automatically matches the generic neural network architecture to a given imaging dataset. The network performance is then optimized, and the final algorithm is generated. Separate portions of the labeled OCT fabric scans are allocated to train and validate the deep learning architecture. The program Vision AutoML runs on cloud and works by using reinforcement learning (RL) and a recurrent neural network (RNN) that specifies the hyperparameters for a model. At the initial step, the RNN will propose a random set of hyperparameter specifications such as layer count, receptive fields, and nodes per layer. The model accuracy is monitored and utilized as a reward/ punishment signal for the RL algorithm that in return renews the RNN parameters. This procedure optimizes the model by rewarding models with high accuracy and punishing those with less accuracy. The theoretical details of how the algorithm works are given in [42].

Additionally, as shown in Figure 2, different independent scans are used to test the deep learning algorithm and analyze results to quantify the performance objectively. In the actual experiment, 147 images were separated by the algorithm randomly and used for the test runs, from which 


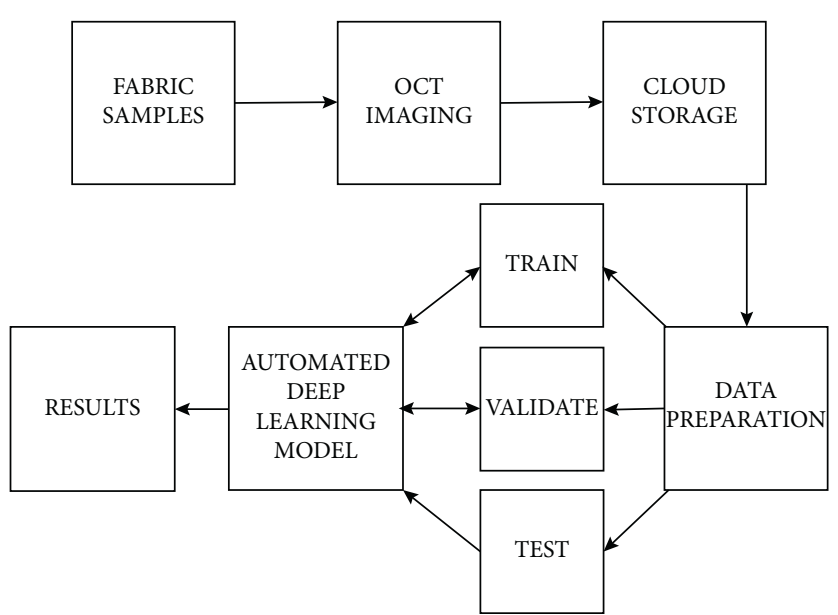

FIGURE 2: Schematics of the automated deep learning procedure. The trained model classifies fabrics made from cotton, polyester, and wool fibers.

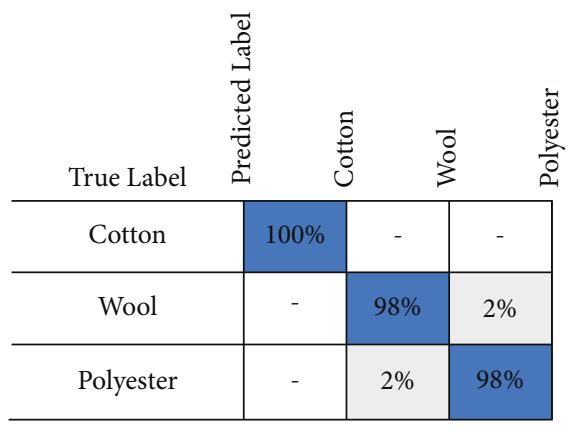

(a)

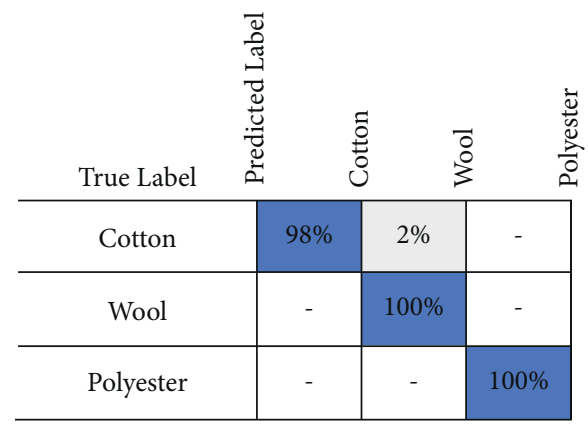

(b)

Figure 3: Confusion matrix for the deep learning model deployed for the (a) cloud and (b) mobile device.

the recall, precision, and confusion matrixes are calculated. Through the Vision AutoML API program, we created two models, one that worked on the cloud and one model that operated on mobile devices. The training times for the cloud and mobile device model were 6 and 0.78 node hours, respectively. The created trained models then can be downloaded from the cloud server.

\section{Results and Discussion}

The automated deep learning models were analyzed by objective metrics used commonly by the artificial intelligence community. Precision, recall, and the confusion matrix of the algorithms were reported since the task investigated in this work fell in the category of classification in machine learning. Precision denotes the fraction of relevant instances among the retrieved instances, meaning that a machine learning model has a precision of $100 \%$ if there are no false positives. The recall is calculated from the fraction of the total amount of relevant instances that were actually recovered. In other words, a model has $100 \%$ recall if it does not have any false negatives. In order to give the insight into the errors made by the deep learning classifier, we present the confusion matrices.
Moreover, we also give the OCT scans corresponding to the false negative and false positive outcomes. Both models had a threshold of 0.5 applied. The first model that was deployed as a cloud-based model had the confusion matrix given in Figure 3(a). The precision and the recall were both 98.64\% indicating that the model could classify fabric fiber material type from OCT scans successively. The model generated for mobile device operation had a $100 \%$ precision and $98.64 \%$ recall. The confusion matrix for this model is given in Figure 3(b).

The results show that the automated deep learning models, after trained with labeled OCT scans corresponding to fabrics woven with wool, cotton, and polyester fibers, can later classify material types successfully with high precision and recall.

In Figure 4, we give the OCT scans corresponding to the false positive and negative results. One can notice that the height of the OCT scan on the right is not optimized, resulting in the saturation in the image, which could have been a reason why the model did not provide the correct outcome for this specific input. This indicates that using high-quality OCT images for the deep learning training and the test plays a decisive role in the quality of the material classification. 

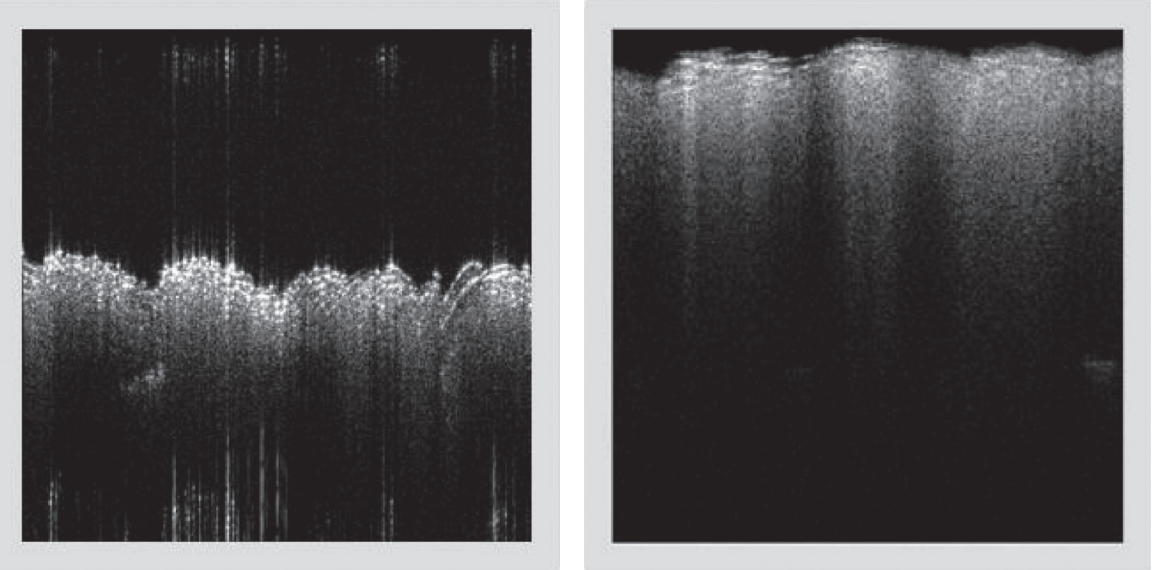

FIGURE 4: The false positive (left OCT scan): the deep learning model incorrectly predicted polyester; false negative results (OCT scan on the right): the model should have predicted polyester.

\section{Conclusions}

In this article, we have successfully demonstrated that the fiber type of a fabric can be identified through OCT imaging and deep learning. Fabrics that were composed of wool, cotton, and polyester fibers were scanned with an OCT device. These scans were used to train automated deep learning models. We demonstrated that the material classification procedure can be done nondestructively using optical coherence tomography (OCT) and deep learning. We presented the recall, precision, and confusion matrixes of the created deep learning models. Based on these results, we conclude that fiber material classification can be achieved nondestructively with high accuracy by OCT imaging and deep learning. Deep-learning-based models can be employed in recycling plants in sorting wool, cotton, and polyester fibers automatically, making manufacturing more sustainable [43]. All the data collected and used in these experiments were uploaded on a data repository. These data can be used to train deep learning networks, test existing machine learning algorithms, or help develop new deep learning systems for automated material classification. With advances in technology, we foresee that optical imaging-based methods combined with artificial intelligence will be used more frequently in the textile industry $[44,45]$.

\section{Data Availability}

The raw image data used to support the findings of this study have been deposited in the figshare repository: OCT image data of cotton-, polyester-, and wool-based fabrics to train and test deep learning algorithms for automated material classification (https://doi.org/10.6084/m9.figshare. 16732591.v1).

\section{Conflicts of Interest}

The authors declare that there are no conflicts of interest regarding the publication of this paper.

\section{References}

[1] A. Basit, W. Latif, M. Ashraf et al., "Comparison of mechanical and thermal comfort properties of Tencel blended with regenerated fibers and cotton woven fabrics," Autex Research Journal, vol. 19, no. 1, pp. 80-85, 2019.

[2] N. Sarwar, U. B. Humayoun, A. A. Khan et al., "Engineering of sustainable clothing with improved comfort and thermal properties-A step towards reducing chemical footprint," Journal of Cleaner Production, vol. 261, Article ID 121189, 2020.

[3] W. Wardiningsih and O. Troynikov, "An evaluation of force attenuation, comfort properties and density of materials for hip protective pads," Journal of Engineered Fibers and Fabrics, vol. 14, 2019.

[4] S. Ahmad, T. Ullah, and Ziauddin, "Fibers for technical textiles," in Fibers for Technical Textiles, pp. 21-47, Springer International Publishing, Cham, Switzerland, 2020.

[5] A. Jabbar, U. Tariq, T. Hussain, A. Basit, A. Hai, and M. Zubair, "Effect of polyester and elastane linear density on the physical and mechanical properties of dual-core-spun cotton yarns," Journal of Natural Fibers, vol. 17, no. 4, pp. 463-471, 2020.

[6] M. Mahbubul Bashar and M. A. Khan, "An overview on surface modification of cotton fiber for apparel use," Journal of Polymers and the Environment, vol. 21, no. 1, pp. 181-190, 2013.

[7] M. Jaffe, A. J. Easts, and X. Feng, "Polyester fibers," in Thermal Analysis of Textiles and Fibers, pp. 133-149, Elsevier, Amsterdam, Netherlands, 2020.

[8] T. Karthik, R. Rathinamoorthy, and P. Ganesan, "Sustainable luxury natural fibers-production, properties, and prospects," in Handbook of Sustainable Luxury Textiles and Fashion, pp. 59-98, Springer Singapore, Singapore, Singapore, 2015.

[9] A. Afzal, U. Zubair, M. Saeed, M. Afzal, and A. Azeem, "Fibres for medical textiles," in Fibers for Technical Textiles, pp. 169-200, Springer International Publishing, Cham, Switzerland, 2020.

[10] T. Bashir, M. Ali, N.-K. Persson, S. K. Ramamoorthy, and M. Skrifvars, "Stretch sensing properties of conductive knitted structures of PEDOT-coated viscose and polyester yarns," Textile Research Journal, vol. 84, no. 3, pp. 323-334, 2014.

[11] R. Nayak, S. Houshyar, A. Khandual, R. Padhye, and S. Fergusson, "Identification of natural textile fibres," in 
Handbook of Natural Fibres, pp. 503-534, Elsevier, Cham, Switzerland, 2020.

[12] P. Khan, N. Abir, M. Rakib, E. M. S. Bhuiyan, and M. R. Howlader, "A review paper on textile fiber identification," IOSR Journal of Polymer and Textile Engineering, vol. 4, no. 2, pp. 14-20, 2017.

[13] H.-F. Yuan, R.-X. Chang, L.-L. Tian, C.-F. Song, X.-Q. Yuan, and X.-Y. Li, "Study of nondestructive and fast identification of fabric fibers using near infrared spectroscopy," Guang $P u$ Xue Yu Guang Pu Fen Xi, vol. 30, no. 5, pp. 1229-1233, 2010.

[14] J. F. Molloy, M. Naftaly, Y. M. Andreev, T. I. Izaak, G. V. Lanskii, and V. A. Svetlichnyi, "Identification of textile fiber by IR and Raman spectroscopy," in Proceedings of the 2014 39th International Conference on Infrared, Millimeter, and Terahertz Waves (IRMMW-THz), Tucson, AZ, USA, September 2014.

[15] M. Mäkelä, M. Rissanen, and H. Sixta, "Machine vision estimates the polyester content in recyclable waste textiles," Resources, Conservation and Recycling, vol. 161, no. 105007, 2020.

[16] Z. Feng, W. Liang, D. Tao, L. Sun, A. Zeng, and M. Song, "CUnet: component unmixing network for textile fiber identification," International Journal of Computer Vision, vol. 127, no. 10, pp. 1443-1454, 2019.

[17] P. Peets, K. Kaupmees, S. Vahur, and I. Leito, "Reflectance FTIR spectroscopy as a viable option for textile fiber identification," Heritage Science, vol. 7, no. 1, 2019.

[18] H. Zhao, Y. Wang, S. Liu, K. Li, and W. Gao, "Spectral reflectance characterization and fiber type discrimination for common natural textile materials using a portable spectroradiometer," Journal of Archaeological Science, vol. 111, no. 105026, 2019.

[19] K. Ylld1z, "Identification of wool and mohair fibres with texture feature extraction and deep learning," IET Image Processing, vol. 14, no. 2, pp. 348-353, 2020.

[20] L. Navone, K. Moffitt, K.-A. Hansen, J. Blinco, A. Payne, and R. Speight, "Closing the textile loop: enzymatic fibre separation and recycling of wool/polyester fabric blends," Waste Management, vol. 102, pp. 149-160, 2020.

[21] P. Senthil Kumar and S. Suganya, "Test methods and identification of recycled polyester," in Textile Science and Clothing Technology, pp. 69-88, Springer Singapore, Singapore, Singapore, 2020.

[22] M. Sabuncu and M. Akdoğan, "Utilizing optical coherence tomography in the nondestructive and noncontact measurement of egg shell thickness," The Scientific World Journal, vol. 2014, Article ID 205191, 4 pages, 2014.

[23] M. Sabuncu and M. Akdoğan, "Photonic imaging with optical coherence tomography for quality monitoring in the poultry industry: a preliminary study," Revista Brasileira de Ciência Avícola, vol. 17, no. 3, pp. 319-324, 2015.

[24] S.-H. Kim, J.-H. Kim, and S.-W. Kang, "Nondestructive defect inspection for LCDs using optical coherence tomography," Displays, vol. 32, no. 5, pp. 325-329, 2011.

[25] X. Shao, X. Chen, X. Yu et al., "Nondestructive measurement of conformal coating thickness on printed circuit board with ultra-high resolution optical coherence tomography," IEEE Access, vol. 7, pp. 18138-18145, 2019.

[26] M. Sabuncu and H. Özdemir, "Recognition of fabric weave patterns using optical coherence tomography," Journal of the Textile Institute, vol. 107, no. 11, pp. 1406-1411, 2016.

[27] M. Sabuncu, H. Ozdemir, and M. U. Akdogan, "Automatic identification of weave patterns of checked and colored fabrics using optical coherence tomography," IEEE Photonics Journal, vol. 9, no. 5, pp. 1-8, 2017.

[28] M. Sabuncu and H. Özdemir, "Recognition of weave patterns of striped fabrics using optical coherence tomography," Fibres and Textiles in Eastern Europe, vol. 26, no. 3(129), pp. 98-103, 2018.

[29] M. Sabuncu and H. Ozdemir, "Contactless measurement of fabric thickness using optical coherence tomography," The Journal of the Textile Institute, 2021.

[30] N. Ghassemi, M. Tayarani Darbandy, A. Shoeibi et al., "Material Recognition for Automated Progress Monitoring Using Deep Learning Methods," 2020, https://arxiv.org/abs/ 2006.16344.

[31] Z. Xiao, X. Liu, J. Wu et al., "Knitted fabric structure recognition based on deep learning," Journal of the Textile Institute, vol. 109, no. 9, pp. 1217-1223, 2018.

[32] W. Puarungroj and N. Boonsirisumpun, "Recognizing handwoven fabric pattern designs based on deep learning," in Advances in Intelligent Systems and Computing, pp. 325-336, Springer Singapore, Singapore, Singapore, 2019.

[33] S. Meng, R. Pan, W. Gao, J. Zhou, J. Wang, and W. He, "A multi-task and multi-scale convolutional neural network for automatic recognition of woven fabric pattern," Journal of Intelligent Manufacturing, vol. 32, no. 4, pp. 1147-1161, 2020.

[34] F. Pereira, V. Carvalho, R. Vasconcelos, and F. Soares, "A review in the use of artificial intelligence in textile industry," in International Conference Innovation in Engineering, pp. 377-392, Springer, Cham, Switzerland, 2021.

[35] L. Faes, S. K. Wagner, D. J. Fu et al., "Automated deep learning design for medical image classification by health-care professionals with no coding experience: a feasibility study," The Lancet Digital Health, vol. 1, no. 5, pp. e232-e242, 2019.

[36] I. Yılmazlar and M. Sabuncu, "Speckle noise reduction based on induced mode Hopping in a semiconductor laser diode by drive current modulation," Optics \& Laser Technology, vol. 73, pp. 19-22, 2015.

[37] I. Yilmazlar and M. Sabuncu, "Implementation of a current drive modulator for effective speckle suppression in a laser projection system," IEEE Photonics Journal, vol. 7, no. 5, pp. 1-6, 2015.

[38] A. G. Türk, M. Sabuncu, S. Ünal, B. Önal, and M. Ulusoy, "Comparison of the marginal adaptation of direct and indirect composite inlay restorations with optical coherence tomography," Journal of Applied Oral Science, vol. 24, no. 4, pp. 383-390, 2016.

[39] A. G. Turk, M. Sabuncu, and M. Ulusoy, "Evaluation of adaptation of ceramic inlays using optical coherence tomography and replica technique," Brazilian Oral Research, vol. 32, pp. 1-10, 2018.

[40] M. Sabuncu and H. Ozdemir, "OCT image data of cotton, polyester and wool based fabrics to train and test deep learning algorithms for automated material classification," Figshare Dataset, 2021.

[41] E. Bisong, "Google automl:cloud vision," in Building Machine Learning and Deep Learning Models on Google Cloud Platform, pp. 581-598, Apress, Berkeley, CA, USA, 2019.

[42] B. Zoph, V. Vasudevan, J. Shlens, and Q. V. Le, "Learning transferable architectures for scalable image recognition," 2018, https://arxiv.org/abs/1707.07012.

[43] A. Goyal and R. Nayak, "Sustainability in yarn manufacturing," in Sustainable Technologies for Fashion and Textiles, pp. 33-55, Elsevier, Amsterdam, Netherlands, 2020. 
[44] C. Giri, S. Jain, X. Zeng, and P. Bruniaux, "A detailed review of artificial intelligence applied in the fashion and apparel industry," IEEE Access, vol. 7, pp. 95376-95396, 2019.

[45] K. Cura, N. Rintala, T. Kampouri, E. Saarimaki, and P. Heeikkila, "Textile recognition and sorting for an automated line using near infrared spectroscopy," Recycling, vol. 6, pp. 6-12, 2021. 